\title{
Medical Data Sharing in Cloud Computing with Privacy Protection and Intrusion Avoidance for Patient
}

\author{
R. Siva Kumar \\ Dept. of Computer Sci. \& Engg. \\ MJR College of Engg \& Tech. \\ Piler, Chittoor,India
}

\author{
K. Suresh \\ Dept. of Computer Sci. \& Engg. \\ MJR College of Engg \& Tech. \\ Piler, Chittoor,India
}

\author{
K. Suresh \\ Dept. of Computer Sci.\& Engg. \\ MJR College of Engg \& Tech. \\ Piler, Chittoor,India
}

\begin{abstract}
With the popularity of smart electronic devices, along with the development of clouds and cloudlet technology, there has been increasing need to provide better medical care. The processing chain of medical data mainly includes data collection, data storage and data sharing, etc. Traditional healthcare system often requires the delivery of medical data to the cloud, which involves users' sensitive information and causes communication energy consumption. Practically, medical data sharing is a critical and challenging issue. Along these lines in this paper, we develop a novel human services framework by using the adaptability of cloudlet. The elements of cloudlet incorporate security assurance, information sharing and interruption location. In the phase of information accumulation, we initially use Number Theory Research Unit (NTRU) technique to scramble client's body information gathered by wearable gadgets. Those information will be transmitted to close-by cloudlet in a vitality productive form. Also, we introduce another trust model to assist clients with selecting trustable accomplices who need to share put away information in the cloudlet. The trust demonstrate additionally causes comparable patients to speak with each other about their illnesses. Thirdly, we partition clients' therapeutic information put away in remote billow of healing facility into three sections, and give them appropriate insurance. At long last, keeping in mind the end goal to shield the medicinal services framework from malignant assaults, we build up a novel cooperative interruption discovery framework (IDS) strategy in view of cloudlet work, which can viably keep the remote social insurance huge information cloud from assaults. Our examinations show the viability of the proposed conspire.
\end{abstract}

Keywords- privacy protection, data sharing, collaborative intrusion detection system (IDS), healthcare.

\section{INTRODUCTION}

With the improvement of human services enormous information and wearable innovation [1], and also distributed computing and correspondence advances, cloud-helped medicinal services huge information registering ends up basic to meet clients' consistently developing requests on wellbeing conference.

However, it is challenging issue to personalize specific healthcare data for various users in a convenient fashion. Past work proposed the blend of interpersonal organizations and medicinal services administration to encourage the hint of the malady treatment process for the recovery of ongoing illness data Healthcare social stage, for example, Patients-LikeMe[2], can acquire data from other comparable patients through information partaking as far as client's own particular discoveries.

In spite of the fact that sharing medicinal information on the interpersonal organization is valuable to the two patients and specialists, the touchy information may be spilled or stolen, which causes security. and security problems without efficient protection for the shared data. Therefore, how to balance privacy protection with the convenience of medical data sharing becomes a challenging issue. In terms of the above problems, this paper proposes a cloudlet based healthcare system. The body data collected by wearable devices are transmitted to the nearby cloudlet. Those data are further delivered to the remote cloud where doctors can access for disease diagnosis.

According to data delivery chain, we separate the privacy protection into three stages. In the first stage, user's vital signs collected by wearable devices are delivered to a closet gateway of cloudlet. During this stage, data privacy is the main concern [2]. In the second stage, user's data will be further delivered toward remote cloud through cloudlets. A cloudlet is formed by a certain number of mobile devices whose owners may require and/or share some specific data contents.

Thus, both privacy protection and data sharing are considered in this stage. Especially, we use trust model to evaluate trust level between users to determine sharing data or not. Considering the users' medical data are stored in remote cloud, we classify these medical data into different kinds and take the corresponding security 
policy. In addition to above three stages based data privacy protection, we also consider collaborative IDS based on cloudlet mesh to protect the cloud ecosystem [3]. In particular in any of the health sector, a sensitive patient record has to be kept confidential.

Security of such delicate data must be ensured, in the event that it is scrambled by the information proprietor before it is being put away in server farms. Subsequently, just the confirmed information proprietor will have the capacity to get to the information by unscrambling it utilizing given private decoding key.

Encryption process confines the likelihood to outsource calculation over the remotely put away information [4], particularly if the server farm have no entrance to the decoding key, since the key is especially fundamental, for any standard encryption plans, to unscramble the information by playing out certain calculation upon it. This system authorizes the physician and medical researcher. The rest of the paper is organized as follows: Section II presents the related work; Section III presents the proposed work; Section and concludes in Section IV.

\section{RLEATED WORK}

This section presents the prior work of the medical data sharing models. The author in [5] has demonstrated that authentication scheme may suffer from different attacks and may fail to provide several security characteristics.

Later, proposed an authenticated key agreement scheme by applying "chaotic map-based cryptography" to solve these problems. This scheme realizes the protection of hospital data transmitted in the open channel and provides confidential protection during the remote diagnosing process, allowing the patient to enjoy the secure and convenient healthcare through the TMIS. Security analysis \& performance analysis has been proved for various attacks and better performance and thus it's more suitable for practical applications in TMIS environments.

In [6], considering the sensitive healthcare information in cloud environments, and proposed in a special data scrambling method for healthcare application, where a tiny part of data is used to scramble the remaining data for the purpose of encryption. This method improves in terms of security performance and practicability. ECG signals from both "MIT-BIH arrhythmia" database and "elf-collected" database are used. Conversion into decimal format is based on a quantization resolution of eight bits.

In [7], introduced a novel system for healthcare professionals to enhance their compliance with best practice and regulations using, Microsoft Kinect sensor ${ }^{\text {re }}$ and smart devices' while protecting patient privacy. A contribution for this study will be registration mechanism for a healthcare professional to explicitly give their system the permission to monitor his/her activities. Multiple Kinect sensors are used for improved tracking accuracy and better coverage for bigger workplaces.

Finally, their system generates alerts through designated smart watch according his or her personal preference. In [8], consider a three tier medical body area network (MBAN): inter-MBAN, intra-MBAN, and beyond MBAN. The intra-MBAN transmits sensors' data to a controller, and in turn transmits them to inter- MBAN tier to an access device like a PDA or tablet device, which is usually connected to a patient's medical database. This access device used as a means of communication for intra-MBAN and beyond-MBAN to uses hospital information systems. This is widely deployed in hospitals places security and privacy violation threats . Results show that this scheme achieves much higher privacy protection, at expense of reduced coverage.

In [9], introduced a cloudlet based healthcare system, where they consider privacy of users' physiological data and efficiency of data transmission. They use NTRU, Number Theory Research Unit for data protection during data transmission to the cloudlet.

To share data in the cloudlet, they use users' similarity and reputation to build a trust model. Based on measured users' trust level, the system finds out whether data sharing is performed. They divide data in remote cloud into various kinds and apply encryption mechanism to protect them respectively.

They also proposed collaborative IDS, intrusion detection system against malicious attacks based on cloudlet mesh to protect the whole healthcare system. In [10], contributed to appeal to Data encryption in healthcare cloud computing environment ${ }^{\text {ec }}$. They suggest a hybrid architecture based on Cryptography as a Service(CaaS) includes the private cloud OpenStack platform. Cryptographic operations control the healthcare cloud clients and they prevail keys in the cloud independent of the cloud provider.

Firstly, they summarize cloud computing for healthcare, and provide survey about important concepts regarding cryptography. Then, they investigate optimized realization of homomorphic encryption, RSA and Elliptic based additive homomorphic encryption, which offers better reporting. Finally, they propose a architecture to solve the privacy problem in healthcare cloud which offers a fast point multiplication, while featuring small code and memory requirements. 


\section{PROPOSED WORK}

This section presents the proposed work of the issues on medical data sharing models. The major objectives of the proposed system are:

- To propose a cloudlet based healthcare system.

- To provide privacy protection enabled data delivery chain.

The proposed model composes of four phases, namely,

1. Patient-In this module, there are $n$ numbers of patient are present. Patient should register before doing some operations. And register user details are stored in user module. After registration successful he has to login by using authorized user name and password. Login successful he will do some operations like Send Appointment Request, Access Request, and Receive Prescription.

2. Doctor-The following are the functionalities of the Doctor phases,

- The doctor should be authorized by the cloud server.

- Only authorized doctor can be view the patient details and access the data.

- They can approve the patient health records like sending prescription to the user.

3. Cloudlet-In this module, the CloudLet has to login by using valid name and password. After login successful he/she can do some operations such as Add Doctor, View all Doctor Information, view Patient, and view the Intruder Detection Details.

4. Intruder-In this Intruder module, we develop the following functionalities:

- View patient records means it is showing only encrypted format

- Try to modify data means alert mail send to patient or cloud let.


Fig.1 Illustration of the system architecture: (a) Privacy protection; (b) Collaborative IDS.
By doing so, the merits achieved are:

- A cloudlet based healthcare system is presented, where the privacy of users' physiological data and the efficiency of data transmissions are our main concern. We use NTRU for data protection during data transmissions to the cloudlet.

- In order to share data in the cloudlet, we use users' similarity and reputation to build up trust model. Based on the measured users' trust level, the system determines whether data sharing is performed.

- We divide data in remote cloud into different kinds and utilize encryption mechanism to protect them respectively.

\section{CONCLUSION}

In this project, we investigated the problem of privacy protection and sharing large medical data in cloudlets and the remote cloud. We developed a system which does not allow users to transmit data to the remote cloud in consideration of secure collection of data, as well as low communication cost. However, it does allow users to send data to a cloudlet, which triggers the data sharing problem in the cloudlet. Firstly, we can utilize wearable devices to collect users' data, and in order to protect users privacy, we use cloudlet mechanism to make sure the transmission of users' data to cloudlet in security. Secondly, for the purpose of sharing data in the cloudlet, we use trust model to measure users' trust level to judge whether to share data or not. Thirdly, for privacypreserving of remote cloud data, we partition the data stored in the remote cloud and encrypt the data in different ways, so as to not just ensure data protection but also accelerate the efficacy of transmission. Finally, we propose collaborative IDS based on cloudlet mesh to protect the whole system. User asks the question to the doctor online and doctor give the answer to user.

\section{REFERENCES}

[1]. Min Chen et al, "Privacy Protection and Intrusion Avoidance for Cloudlet-based Medical Data Sharing", IEEE transactions on Cloud computing, 2017.

[2]. R. Mitchell and I.-R. Chen, "Behavior rule specification-based intrusion detection for safety critical medical cyber physical systems," Dependable and Secure Computing, IEEE Transactions on, vol. 12, no. 1, pp. 16-30, 2015.

[3]. Y. Shi, S. Abhilash, and K. Hwang, "Cloudlet mesh for securing mobile clouds from intrusions and network attacks," in The Third IEEE International Conference on Mobile Cloud Computing, Services, and Engineering,(Mobile Cloud 2015).

[4]. Tadapaneni, N. R. (2017). Different Types of Cloud Service Models. Available at SSRN 3614630. 
[5]. M. Quwaider and Y. Jararweh, "Cloudlet-based efficient data collection in wireless body area networks," Simulation Modelling Practice and Theory, vol. 50, pp. 57-71, 2015.

[]. M. S. Hossain, "Cloud-supported cyber-physical localization framework for patients monitoring," 2015.

[6]. J. Zhao, L. Wang, J. Tao, J. Chen, W. Sun, R. Ranjan, J. Kołodziej, A. Streit, and D. Georgakopoulos, Journal of Computer and System Sciences, vol. 80, no. 5, pp. 994-1007, 2014.

[7]. Tadapaneni, N. R. (2018). Cloud Computing: Opportunities And Challenges. International Journal of Technical Research and Applications.

[]. H. Mohamed, L. Adil, T. Saida, and M. Hicham, "A collaborative intrusion detection and prevention system in cloud computing," in AFRICON, 2013. IEEE, 2013, pp. 1-5.

[8]. R. Zhang and L. Liu, "Security models and requirements for healthcare application clouds," in Cloud Computing (CLOUD), 2010 IEEE .

[9]. K. Hung, Y. Zhang, and B. Tai, "Wearable medical devices for telehome healthcare," in Engineering in Medicine and Biology Society, 2004. IEMBS' $04.26^{\text {th }}$ Annual International Conference of the IEEE, vol. 2. IEEE, 2004, pp. 5384-5387.

[10]. Guiwu, W. Y. (2018, June). Research on Genetic Algorithm for Resource Scheduling in Cloud Computing Based on User Satisfaction. In 5th International Conference on Electrical \& Electronics Engineering and Computer Science (ICEEECS 2018) (Vol. 5).

[11]. Patel, A., \& Tiwari, P. (2018). Cloud Computing Security, Privacy Improvements Using Virtualized High Trust Zone. International Journal of Modern Trends in Engineering and Science, 3(12). 